\title{
Análise espaço-temporal da epidemia do HIV em idosos num estado amazônico brasileiro
}

\author{
Spatiotemporal analysis of the HIV epidemic in older people in a Brazilian Amazon state
}

Thayse Moraes de Moraes' (ID Wellingtton Augusto Andrade Fernandes ${ }^{2}$ (D) Carlos Jaime Oliveira Paes ${ }^{3}$ ID Glenda Roberta Oliveira Naiff Ferreiral Lucia Hisako Takase Gonçalves' Eliã Pinheiro Botelho' $\mathbb{D}$

\section{Resumo}

Objetivo: Analisar espaço-temporalmente a incidência do vírus da imunodeficiência humana (HIV) e da síndrome da imunodeficiência adquirida (Aids) entre idosos no estado do Pará, Brasil, nos anos de 2007 a 2018. Método: Estudo ecológico utilizando notificações de casos de HIV/Aids em idosos provenientes do Sistema de Informação de Agravos de Notificação. A taxa de incidência do HIV/Aids foi analisada temporalmente pelo método de joinpoint e espacialmente pelas técnicas de autocorrelação de Moran, de varredura e de regressão espacial. Resultados: 2.639 notificações de HIV/Aids foram elegíveis para o estudo, sendo 1.725 (65,4\%) em homens e 914 (34,6\%) em mulheres. No período do estudo houve aumento de $2.422,5 \%$ na taxa de incidência de HIV nos homens e de 1.929,8\% nas mulheres, sendo o oposto, observado para a taxa de incidência de Aids que aumentou $77,6 \%$ nas mulheres e 40,7\% nos homens. O método joinpoint revelou tendência crescente para a taxa de incidência de HIV (APC $=30 \%, p=0,00)$ e de estabilidade para taxa de incidência de Aids (APC $=3,0 \%, p=0,2$ ). Os municípios mais impactados pela epidemia do HIV foram os do meridional sudeste paraense com moderada associação $\left(R^{2}=0,65\right)$ ao seu crescimento populacional. A análise de varredura espaço-temporal apontou Belém como zona de risco para o HIV/Aids (RR=3,93, $p=0,00 ; 2017-2018)$ Conclusão: Enquanto a incidência de Aids em idosos paraenses permaneceu estável no período de 2007 a 2018 , a de HIV tendeu a crescer. O maior impacto da epidemia ocorreu nos municípios do sudeste paraense com associação ao crescimento populacional, e Belém apresentou risco espaço-temporal para o HIV/Aids.

\footnotetext{
1 Universidade Federal do Pará, Programa de Pós-Graduação em Enfermagem. Belém, PA, Brasil.

2 Universidade Federal do Pará, Núcleo de Altos Estudos Amazônicos, Laboratório de Análises Espaciais (LAENA). Belém, PA, Brasil.

3 Universidade do Estado do Pará, Faculdade de Medicina - Campus Marabá. Marabá, PA, Brasil.
}

Financiamento: Programa Nacional de Cooperação Acadêmica (PROCAD) - Amazônia 2018/CAPES. Processo no. 1699/2018 /88881.200527/2018-01. Bolsa Coordenação de Aperfeiçoamento de Pessoal de Nivel Superior (CAPES).

Os autores declaram não haver conflito na concepção deste trabalho.

Palavras-chaves: Análise Espacial. HIV. Saúde do Idoso. 


\section{Abstract}

Objective: To analyze spatiotemporally the incidence of the human immunodeficiency virus (HIV) and acquired immunodeficiency syndrome (AIDS) among older people in the State of Pará, Brazil, from 2007 to 2018. Method: An ecological study of HIV/AIDS case notifications in older people from the Brazilian Information System on Notifiable Diseases. The HIV/AIDS incidence rate was temporally analyzed by the joinpoint method, and spatially by the Moran autocorrelation of scanning and spatial regression techniques. Results: 2,639 notifications of HIV/AIDS were eligible for the study, with $1,725(65.4 \%)$ being in men and 914 (34.6\%) in women. During the study period, there was an increase of $2,422.5 \%$ in the HIV incidence rate in men and $1,929.8 \%$ in women, with the opposite being observed for the AIDS incidence rate, which increased $77.6 \%$ in women and $40.7 \%$ in men. The joinpoint method showed an increasing trend for the HIV incidence rate (APC $=30 \%, p=0.00$ ) and stability for the AIDS incidence rate (APC $=3.0 \%, p=0.2$ ). The most impacted municipalities by the HIV epidemic were those in the south-eastern part of Pará, with a moderate association $\left(\mathrm{R}^{2}=0.65\right)$ with its population growth. The spatiotemporal scanning analysis pointed to Belém as a risk zone for HIV/AIDS (RR=3.93, $p=0.00 ; 2017-2018$ ). Conclusion: While the incidence of AIDS among older people from Pará remained stable from 2007 to 2018, that of HIV tended to grow. The greatest impact of the epidemic occurred in southeastern Pará municipalities, and it was associated with the population growth; Belém presented a spatiotemporal risk for HIV/AIDS.

\section{INTRODUÇÃO}

No mundo inteiro cerca de 38 milhões de pessoas estão vivendo com o vírus da imunodeficiência humana (HIV). Embora progressos tenham sido alcançados no seu combate, 1,7 milhão de pessoas foram diagnosticadas com o vírus somente no ano de $2019^{1}$. Nesse cenário, destacam-se as pessoas a partir de 50 anos de idade em que vem crescendo o número de novos casos notificados. Anualmente, cerca de 100 mil pessoas nessa faixa etária são diagnosticadas com o HIV em países em desenvolvimento ${ }^{2}$. Porém, tal fenômeno também ainda ocorre em países desenvolvidos. No Canadá, entre 2008 e 2017, a proporção de novos diagnósticos de HIV em pessoas a partir de 50 anos de idade saltou de $15,1 \%$ para $22,8 \%$ de todos os casos notificados ${ }^{3}$. O mesmo aconteceu na União Europeia com a taxa de notificação de HIV (x100 mil habitantes), entre 2004 e 2015, aumentando na mesma faixa etária de 3,5 para 4,8 nos homens e de 1 para 1,2 nas mulheres ${ }^{4}$. Nos Estados Unidos, em 2018, a cada seis diagnósticos de HIV, um deles era em pessoa acima de 50 anos de idade 5 . No Brasil, do total de novos casos notificados de HIV/Aids em 2008, a faixa etária de 50 anos representava $9,34 \%$, passando para $12,42 \%$ em $2018^{6}$.
A maioria dos estudos sobre a epidemia do HIV trata de jovens e adolescentes. No entanto, embora a atividade sexual diminua com a idade, muitos idosos permanecem sexualmente ativos, com baixo conhecimento sobre as formas de transmissão e prevenção do HIV e subestimando o risco de infecção, o que os torna mais vulneráveis ${ }^{7}$. Diante desse quadro, preocupa o fenômeno mundial do envelhecimento populacional. Globalmente, estimase que em 2050 pessoas acima de 65 anos de idade representem $16 \%$ de toda a população mundial ${ }^{9}$.

Para combater a epidemia do HIV, deve-se considerar sua dinamicidade espacial por estar diretamente influenciada por fatores sociopolíticos e econômicos ${ }^{10}$. Nesse contexto, os estudos de análise espacial destacam-se por permitirem a identificação de áreas com as maiores pressões epidemiológicas e a associação com fatores territoriais. Já os estudos de análise temporal permitem avaliar o impacto anual de políticas públicas voltadas para o combate à epidemia ${ }^{11}$. Somente três estudos de análise espacial envolveram as pessoas de 50 anos em diante: dois na China ${ }^{12,13}$ e outro no Brasil ${ }^{14}$. Na China houve maior incidência do HIV/Aids em pessoas a partir de 50 anos de idade em províncias pertencentes ao circuito do tráfico de drogas ilícitas ${ }^{12,13}$. No estado 
da Paraíba, Brasil, foi observada maior incidência de HIV/Aids nos municípios com população superior a 100 mil habitantes, com a epidemia se expandindo nos municípios costeiros e para o interior do estado ${ }^{14}$.

Embora a taxa média de detecção do HIV/Aids no Brasil tenha decrescido entre 2008 e 2018, na região Norte do Brasil aumentou 21,8\%. Em 2019, o Pará foi classificado como quinto estado brasileiro com a maior taxa de detecção do HIV/Aids, e Belém, sua capital, como a segunda capital entre as demais capitais brasileiras. Entre os 100 municípios brasileiros com mais de 100 mil habitantes e maiores taxas de detecção do HIV/Aids, Belém, Marituba e Ananindeua ocupam o segundo, terceiro e nono lugar, respectivamente ${ }^{1}$. Além da baixa cobertura da Estratégia de Saúde da Família $(\mathrm{ESF})^{15}$, no Pará existem somente 32 unidades dispensadoras de antirretrovirais (ARV) para os seus 144 municípios (http://azt.aids.gov.br), das quais somente duas localizadas em Belém fornecem a Profilaxia Pré-Exposição (PreP) ${ }^{1}$.

Mesmo com diversificada fauna e recursos

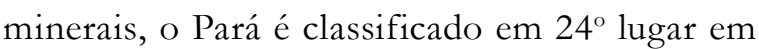
termos de Índice de Desenvolvimento Humano (IDH) entre as 27 unidades federativas do Brasil ${ }^{16}$. Além do baixo investimento em infraestrutura urbana e baixa cobertura de $\mathrm{ESF}^{15,17}$, os municípios do interior paraense passam por um processo de urbanização acelerada devido ao desenvolvimento da indústria extrativista mineral, atraindo muitos migrantes em busca de emprego e melhores condições de vida ${ }^{18}$, cenário propício à transmissão das infecções sexualmente transmissíveis (IST).

O objetivo do presente estudo foi analisar a epidemia do HIV entre pessoas idosas no Pará, Brasil, por meio do uso de técnicas de análise espacial e temporal.

\section{MÉTODOS}

Estudo ecológico realizado com dados secundários provenientes do Sistema de Informações de Agravos de Notificação (SINAN) fornecidos pela Secretaria de Saúde do Estado do Pará (SESPA).

O Pará é o segundo estado brasileiro com maior área territorial $\left(1.245 .870,798 \mathrm{Km}^{2}\right)$ e população de
8.175.156 pessoas, estimando-se que o estrato etário acima de 50 anos é superior a 1.231.570 habitantes, representando $15,06 \%$ da população total ${ }^{19}$.

Foram consultados os registros de HIV e Aids entre pessoas de 50 anos ou mais, notificados ao Sistema de Informação de Agravos de Notificação (SINAN) e fornecidos pela SESPA. Segundo o Programa Conjunto das Nações Unidas (UNAIDS), toda a pessoa vivendo com o HIV a partir de 50 anos de idade é considerada idosa ${ }^{20}$. Somente as notificações com endereços de residência no Pará foram incluídas no estudo, excluindo-se as informações em duplicidade e aquelas sem município de residência.

A coleta de dados se deu no período de janeiro a fevereiro de 2020. Foram coletados dados das seguintes variáveis: idade, sexo, data do diagnóstico, tipo de diagnóstico (HIV ou Aids) e município de residência. Para calcular a incidência anual municipal do HIV/Aids dividiu-se o número de notificações pela população de pessoas a partir de 50 anos de idade, multiplicando-se o resultado por 100.000 . Empregaram-se aqui as projeções populacionais para cada ano obtidas no site do Instituto Brasileiro de Geografia e Estatística (IBGE). Para obter o percentual de crescimento populacional, subtraiuse a projeção populacional de 2018 da projeção de 2007. O resultado foi, então, dividido pela projeção populacional de 2007 e multiplicado por 100.

\section{Análise temporal}

Embora o diagnóstico do HIV tenha passado a ser de notificação compulsória somente a partir de $2014^{21}$, na análise temporal as taxas de incidência do HIV e de Aids foram analisadas separadamente, de forma a verificar o comportamento da taxa de incidência de cada um deles em separado. As taxas anuais de incidência foram submetidas à regressão temporal pelo modelo de Joinpoint ${ }^{22}$ para calcular a variação percentual anual (APC, do inglês annual percent change). O APC é calculado com base nos pontos de inflexão numa série histórica em que linhas de múltiplos segmentos são testadas para verificar se explicam melhor o comportamento da série do que somente uma linha reta. $\mathrm{O}$ valor da tendência anual é calculado com base na união das linhas retas 
em escala logarítmica. A tendência é considerada crescente ou drescente se o APC for positivo ou negativo, respectivamente, e valor de $p \leq 0,05$. Caso contrário, a tendência é considerada estacionária.

\section{Distribuição e autocorrelação espacial da taxa de incidência do HIV/Aids}

Para a análise espacial foi empregada a incidência do HIV/Aids por quadriênio (2007-2010, 20112014, 2015-2018) de modo a evitar as flutuações anuais. A taxa de incidência foi calculada tomando por base a média populacional de cada município para cada um dos quadriênios. Isso feito, analisouse a distribuição espacial da taxa de incidência e empregou-se a autocorrelação espacial de Moran global. O índice de Moran global $(I)$ varia de -1 a 1 , sendo que com valores negativos e $p$-valor $\leq 0,05$ aponta a autocorrelação inversa, município com alta taxa de incidência circundado por municípios com baixa taxa de incidência, ou vice-versa (agrupamento alta-baixa ou baixa-alta incidênca, respectivamente); quando o valor de I é positivo e $p$-valor $\leq 0,05$, ocorre a autocorrelação direta (agrupamento baixa-baixa e alta-alta incidência). Em caso contrário, considera-se distribuição aleatória. Ambas as análises foram feitas empregando o software Arcgis (10.6.1).

A análise de Moran global aponta se há, ou não, agregação espacial. Porém, não mostra a localização dos agrupamentos. Para isso, empregou-se a análise de Moran local através do método de indicador local de associação espacial (LISA), com a matriz de primeira ordem de contiguidade do tipo rainha e o p-valor obtido através de 999 permutações.

\section{Análise de varredura espacial}

Com o intuito de identificar as áreas de risco para a epidemia do HIV aplicou-se a varredura espacial utilizando o software SaTScan (Versão 9.6.1), método proposto por Kulldorff and Nagarwalla ${ }^{23}$. Em resumo, para o cálculo dos aglomerados espaciais, o SaTScan emprega janelas circulares ou elípticas, enquanto para os agrupamentos espaço-temporais as janelas são elípticas com base circular, a base correspondendo ao espaço, e a altura, ao tempo. As janelas movem-se no espaço e no tempo em busca dos agrupamentos de risco comparando sempre o risco dentro e fora da janela. Para cada alteração encontrada, o programa calcula a razão de verossimilhança (LLR) e o valor de significância estatística através das permutações de Monte Carlo. A janela com a maior LLR e com $p \leq 0,05$ é considerada um agrupamento de risco. Para identificar os aglomerados de risco espaciais, em nosso estudo foi empregado o modelo discreto de Poison considerando-se agrupamentos circulares, não sobrepostos geograficamente, com o tamanho máximo de cada cluster não ultrapassando $50 \%$ da população exposta, e 999 permutações de Monte Carlo. Já para identificar agregados espaço-temporais, além dos critérios já mencionados, empregou-se a precisão temporal em ano e aglomerado com no máximo $50 \%$ para os doze anos do estudo. A força do agrupamento estimada foi expressa através do cálculo do risco relativo (RR). Considerou-se somente os $\mathrm{RR} \operatorname{com} p \leq 0,05$, em que $\mathrm{RR} \geq 1$ apontava zonas de risco, enquanto aqueles com $\mathrm{RR}<1$ considerados zonas de proteção, ou seja, de menor risco para que o evento ocorresse. Os mapas temáticos contendo os RR foram gerados no software Arcgis (10.6.1).

\section{Análise de regressão espacial}

Para analisar a regressão espacial considerou-se como variável dependente a incidência de HIV/ Aids para todo o período estudado e como variáveis independentes os Índices de Desenvolvimento Humano dos municípios, assim como o percentual de crescimento populacional entre os anos 2007 e 2017, além da taxa de cobertura de ESF. Primeiramente, foi aplicada a análise de correlação de Pearson para verificar a colinearidade entre as variáveis independentes no software IBM SPSS ${ }^{\circledR}$, versão 23. Em seguida, aplicou-se a técnica stepwise no software Geoda (versão 1.14.0) para obter o melhor modelo dos mínimos quadrados ordinários (OLS). Foi evidenciado como o melhor modelo aquele com menor valor de Akaike (Aic), maior $\mathrm{R}^{2}$ e $\mathrm{R}^{2}$ ajustado, fator de inflação da variância (VIF) menor que $10 \mathrm{e}$ o menor $p$-valor. Em seguida, aplicou-se a análise de Moran global para descartar a dependência espacial dos resíduos do modelo no software Arcgis e, só então, os resíduos foram analisados empregando a regressão ponderada geográfica (GWR) com o raio adaptativo 
de Kernel para melhor ajuste ao modelo escolhido. Ao final testou-se novamente a dependência espacial dos resíduos do modelo final do GWR, seguido da elaboração dos mapas corolépticos para visualizar as correlações.

Todos os mapas foram gerados no sistema de coordenadas geográficas Datum Horizontal SIRGAS-2000, sistema de projeção longlat EPSG 4674. Foram considerados de significância estatística todos os resultados $\operatorname{com} p \leq 0,05$.

Este estudo faz parte do macroprojeto de pesquisa "Diagnóstico Situacional das Infecções Sexualmente Transmissíveis no Contexto Amazônico: Análise Geoespacial, Rastreio e Desenvolvimento de Tecnologias Cuidativo-Educacionais" e foi aprovado pelo Comitê de Ética em Pesquisa do Instituto de Ciências da Saúde da Universidade Federal do Pará, sob número de parecer 3.488.663.

\section{RESULTADOS}

De 2007 a 2018 foram notificados ao SINAN 2.679 casos de HIV/Aids em pessoas com 50 anos ou mais no Pará, dos quais foram excluídas 40 notificações devido a incompletudes nas fichas de notificação. Dentre os 2.639 casos elegíveis, 1.725 $(65,4 \%)$ foram em homens e 914 (34,6\%) em mulheres. Durante o período do estudo houve aumento de 2.203,85\% na taxa de incidência de HIV (2007: 0,78, 2018: 17,97) e de 48,50\% na de Aids (2007: 7,71; 2018: 11,45). Analisando separadamente por sexo, a taxa de incidência do HIV aumentou 2.422,5\% nos homens e $1.929,8 \%$ nas mulheres (Homens: $2007=0,89,2018=22,45$; Mulheres: $2007=0,67$. $2018=13,6$ ). Porém, na taxa de incidência de Aids as mulheres apresentaram $77,57 \%$ de aumento e os homens 40,69\% (Homens: $2007=11,63,2018=16,29$; Mulheres: $2007=3,79,2018=6,73)$.

A Tabela 1 mostra a análise de regressão temporal pelo método de joinpoint das taxas anuais de incidência para toda a população a partir de 50 anos e separadas por sexo: masculino e feminino. Tanto para a população em geral, como para o sexo masculino e para o feminino, a taxa de incidência de Aids tendeu à estabilidade durante todo o período do estudo, enquanto a taxa de incidência do HIV tendeu a crescer.

A Figura 1 mostra os mapas da distribuição espacial da incidência do HIV/Aids, em que os municípios do meridional sudeste e do sudoeste paraense apresentam as maiores taxas. Embora a análise global de Moran não tenha apresentado significância estatística em nenhum dos quadriênios avaliados (2007-2010: $I=-0,18, p=0,80 ; 2011-2014: I=$ $0,11, p=0,67 ; 2015-2018: I=0,05, p=0,27)$, a análise de Moran local apontou no quadriênio de 2011-2014 um agrupamento baixa-baixa incidência no meridional sudoeste (Altamira, Itaituba e Novo Progresso) e outro agrupamento alta-alta incidência no sudeste (São Félix do Xingu, Canaã dos Carajás, Parauapebas, Marabá e Ourilândia do Norte). No quadriênio 2015-2018 houve somente um agrupamento altaalta incidência formado pelos municípios Altamira, Itaituba, Novo Progresso, São Félix do Xingu, Canaã dos Carajás, Parauapebas e Marabá.

Tabela 1. Análise de regressão temporal das Taxas de incidência por gênero, para o HIV e Aids em pessoas a partir de 50 anos de idade no Pará, período de 2007 a 2018. Pará, 2020.

\begin{tabular}{lcccccc}
\hline Período & \multicolumn{5}{c}{ HIV } & \multicolumn{2}{c}{ Aids } \\
\cline { 2 - 6 } & APC & $($ IC95\%) & $p$-valor & APC & $($ IC95\%) & $p$-valor \\
\hline $\begin{array}{l}\text { Masculino/Feminino } \\
2007-2018\end{array}$ & 30 & $(22,4-38,1)$ & $p<0,001$ & 3 & $(-2.1-8.4)$ & 0,2 \\
Masculino & & & & & 0,2 \\
$2007-2018$ & 31,8 & $(21,7-42,8)$ & 0,04 & 3,2 & $(-2,3-9,1)$ & 0,3 \\
Feminino & & & & $(-2.8-8.4)$ & 0,3 \\
$2007-2018$ & 24,7 & $(18,6-31)$ & $p<0,001$ & 2,7 & & \\
\hline
\end{tabular}



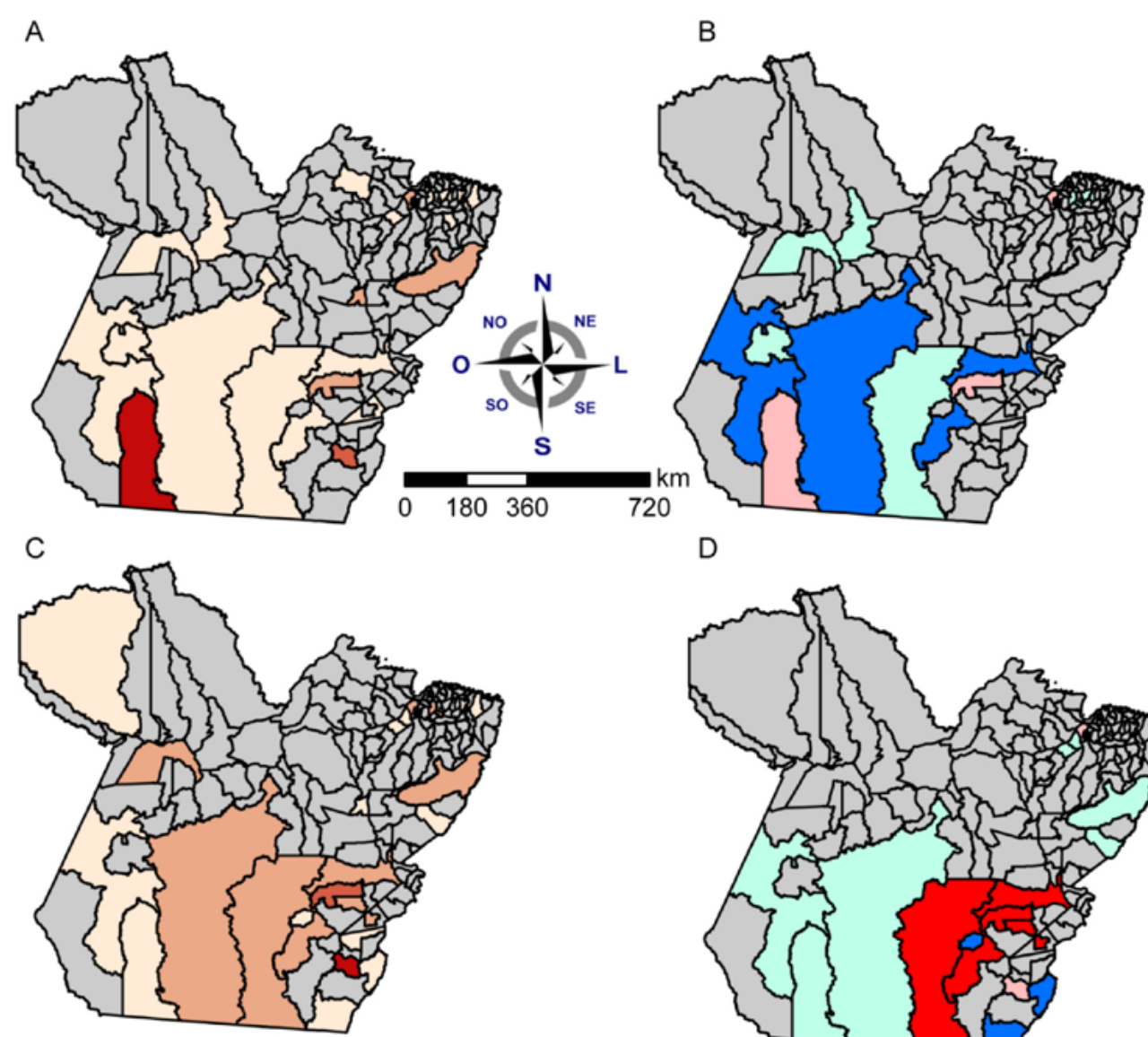

D

E
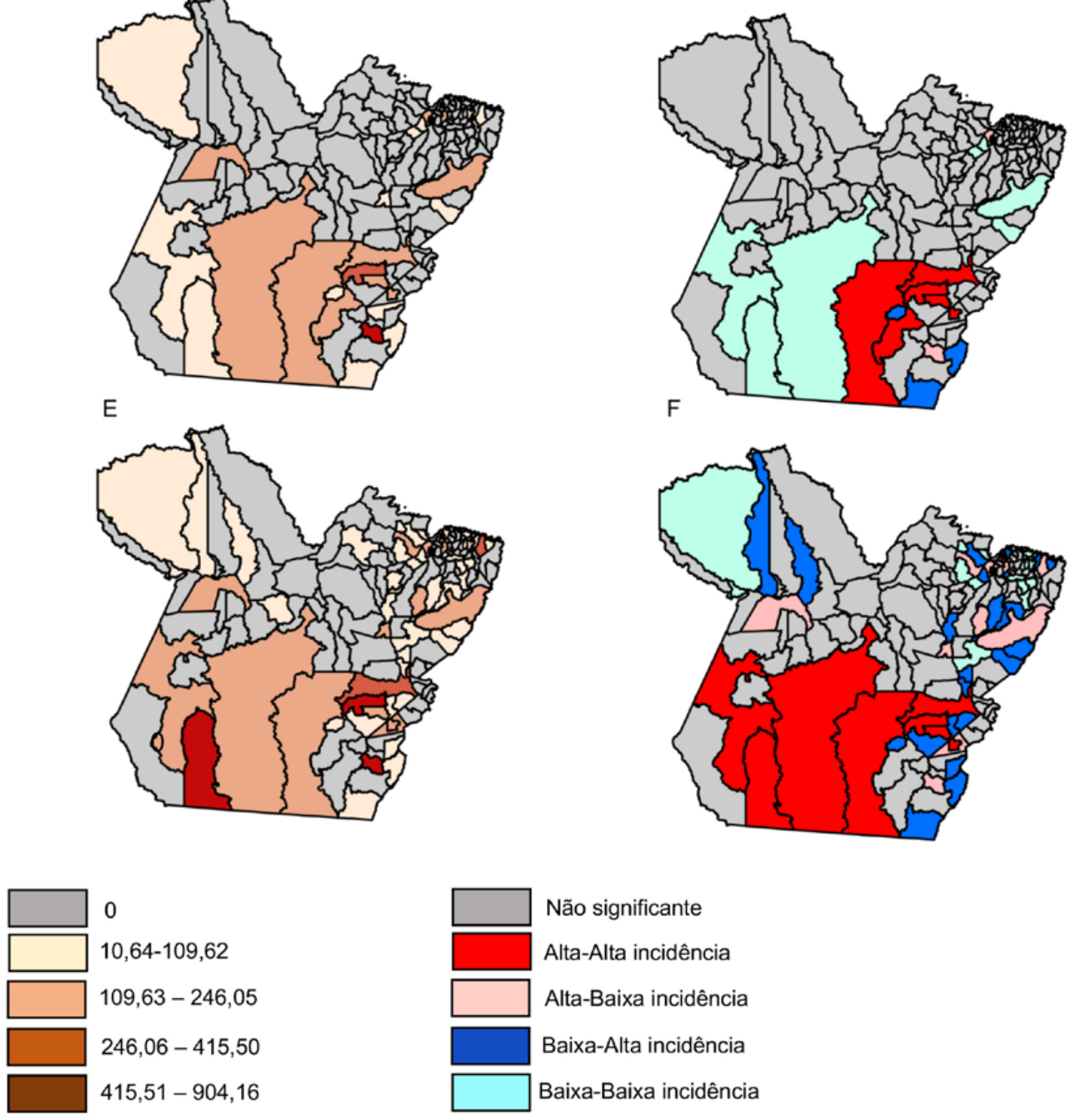

A, C, E: Distribuição espacial da taxa de incidência do HIV/Aids; B, D, F: MAPAS LISA; A,B: 2007-2010; C, D: 2011-2014; E,F: $2015-2018$.

Figura 1. Distribuição espacial da taxa de incidência do HIV/Aids. Pará, 2020. 
A análise de varredura evidenciou duas regiões de risco espacial para o HIV/Aids, sendo o maior $(\mathrm{RR}=2,73, p=0,00)$ observado nos municípios do meridional sudoeste (São Félix do Xingu, Tucumã, Canaã dos Carajás, Parauapebas, Marabá, Ourilândia do Norte, Xinguara, Rio Maria, Redenção, Pau D'Arco, Bannach e Água Azul do Norte) e o outro no município de Belém ( $\mathrm{RR}=2,69, p=0,00)$. Já a varredura espaço-temporal apontou somente Belém como o município de risco no período de 2017 a $2018(\mathrm{RR}=3,93, p=0,00)$ (Figura 2).

Para a regressão espacial, a correlação de Pearson revelou significância estatística entre a variável dependente (incidência do HIV/Aids) com as variáveis crescimento populacional percentual $(\mathrm{r}=0,028, p=0,00)$ e com o IDH-m $(\mathrm{r}=0,41, p=0,00)$.
Porém, na construção do modelo OLS, foi escolhido o modelo explicativo crescimento populacional percentual por apresentar VIF menor que 10 $(\mathrm{VIF}=1,40)$ (Tabela 2).

A análise de GWR mostrou-se o melhor modelo explicativo do modelo 2 do que a do OLS, explicando $65 \%$ do modelo escolhido $\left(\mathrm{R}^{2}=0,65, \mathrm{R}^{2}\right.$ ajustado=0,51, e $\mathrm{AIC}=1845,73)$, com os resíduos gerados não mostrando autocorrelação espacial $(\mathrm{I}=0,04, p=0,46)$. A Figura 3C mostra a correlação direta dos coeficientes da GWR com os maiores índices observados nos municípios do meridional sudeste paraense (Cumaru do Norte, Santa Maria das Barreiras, Santana do Araguaia, Bannach, Redenção, Concórdia do Pará, Santana do Araguaia, Rio Maria).
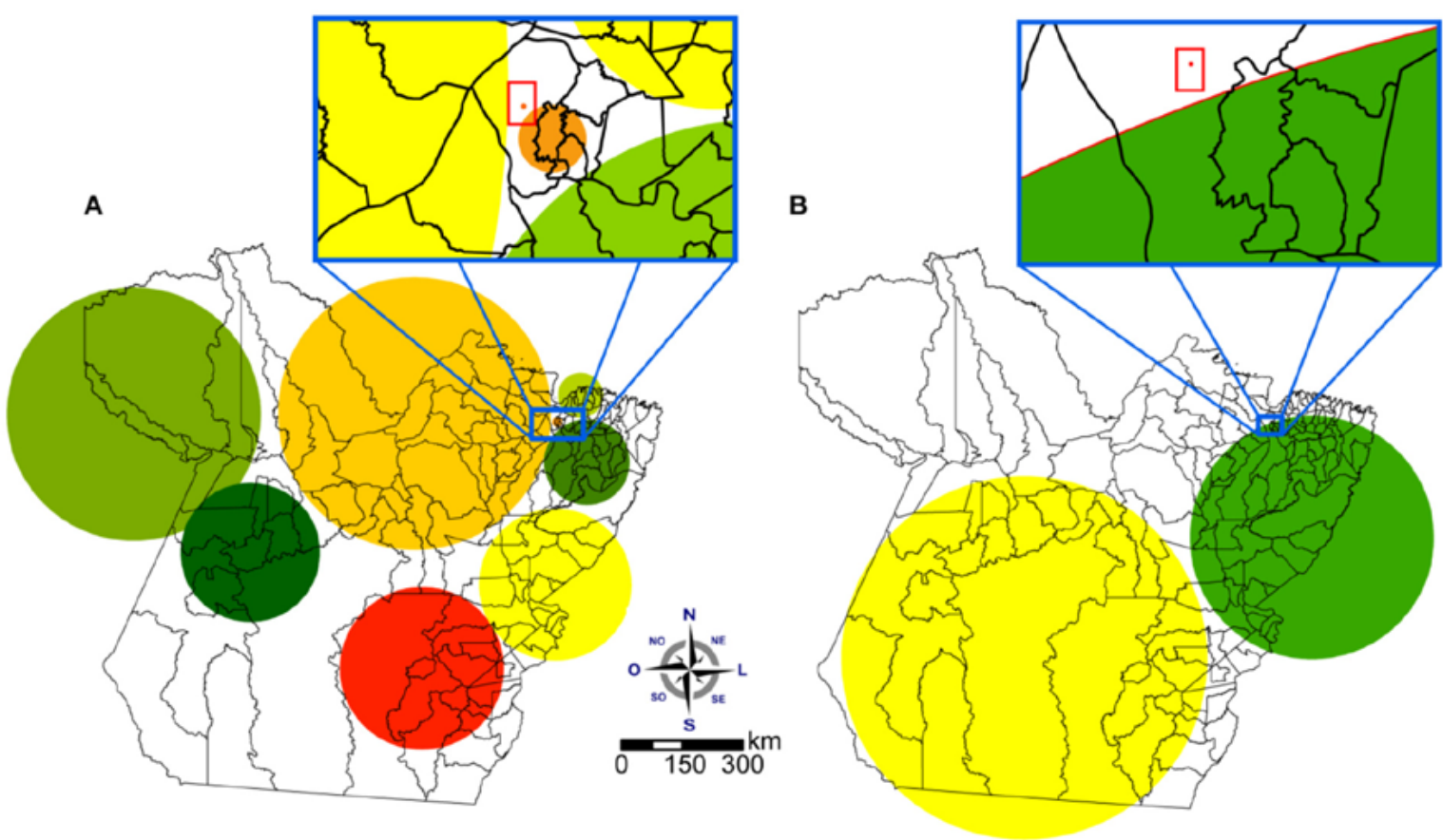

$\mathrm{RR}=0,048 ; p=0,000$ $\mathrm{RR}=0,092 ; p=0,000$ $\mathrm{RR}=0,095 ; p=0,000$ $\mathrm{RR}=0,097 ; p=0,000$ $\mathrm{RR}=0,15 ; p=0,000$
$\mathrm{RR}=0,35 ; p=0,000$

$\mathrm{RR}=0,52 ; p=0,000$

$\mathrm{RR}=2,69 ; p=0,000$

$\mathrm{RR}=2,73 ; p=0,000$
$\mathrm{RR}=0,0003 ; p=0,000 ; 2017-2018$

$\mathrm{RR}=0,003 ; p=0,000 ; 2017-2018$

$\mathrm{RR}=3,93 ; p=0,000 ; 2017-2018$

(A) Risco espacial; (B) Risco espaço-temporal para o HIV/Aids; O retângulo vermelho delimita o círculo indicando Belém como região de risco espacial e espaço-temporal para o HIV/Aids.

Figura 2. Análise de varredura espacial para o HIV/Aids. Pará, 2020. 
Tabela 2. Regressão linear múltipla (OLS) do percentual de crescimento populacional municipal e do IDH-m com a taxa de incidência de HIV/Aids, Pará, 2020.

\begin{tabular}{llll}
\hline Variáveis & Estimativa & Erro Padrão & $p$-Valor \\
\hline Modelo 1 & & & \\
Constante & $-551,15$ & 160,15 & $p<0,001$ \\
Crescimento Populacional percentual & 1067,25 & 277,77 & $p<0,001$ \\
IDH-m & 0 & 0 & 0,08 \\
& $\mathrm{R}^{2}: 0,17 ; \mathrm{R}^{2}$ Ajustado: 0,16; AIC: 1889,$71 ;$ Multicolinearidade: 23,91 & \\
\hline
\end{tabular}

Modelo 2

$\begin{array}{llll}\text { Constante } & 61,51 & 15,63 & p<0,001 \\ \text { Crescimento populacional percentual } & 0,01 & 0 & p<0,001\end{array}$

$\mathrm{R}^{2}$ : 0,08; $\mathrm{R}^{2}$ Ajustado: 0,07; AIC: 1 902,05; Multicolinearidade: 1,40

Modelo 3

Constante

$-658,88$

148,85

$p<0,001$

IDH-m

0,01

0

$p<0,001$

R² Múltiplo: 0,15; R² Ajustado: 0,14; AIC: 1 890,79; Multicolinearidade: 20,89 
A

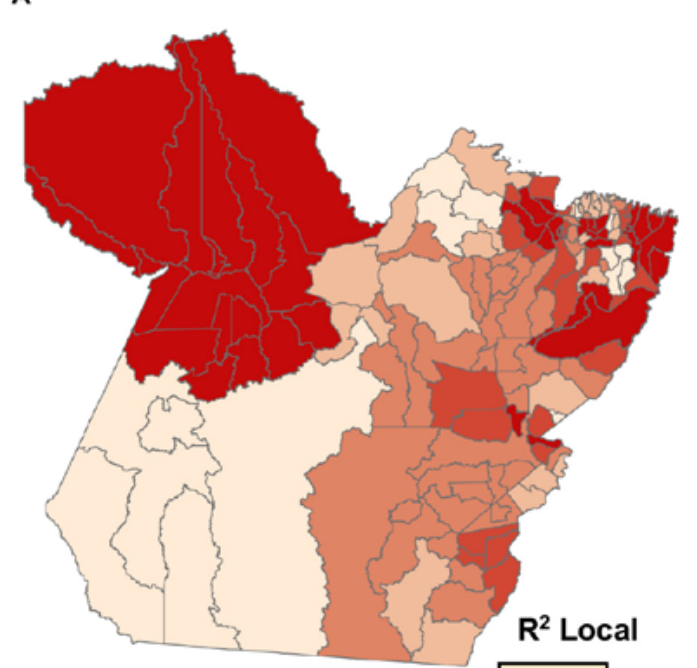

B

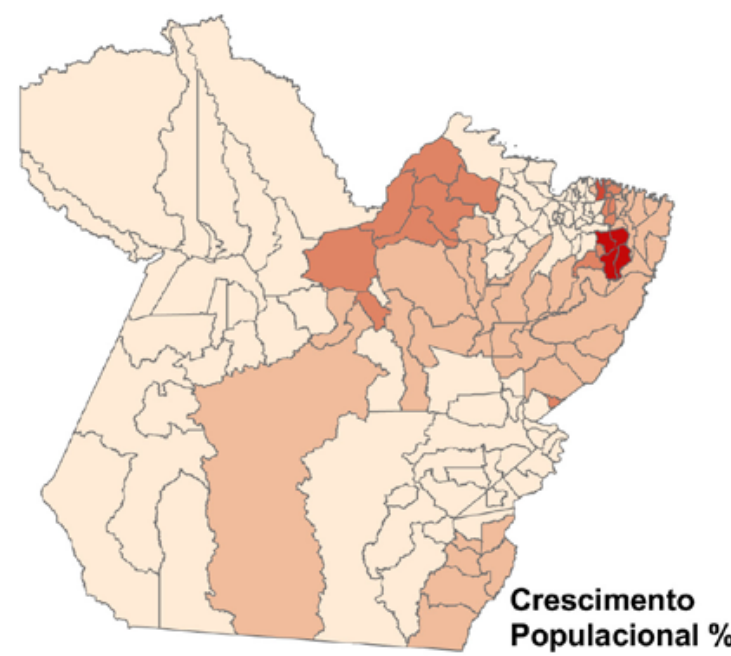

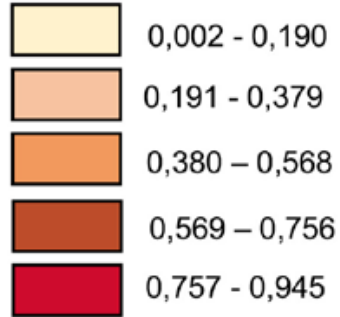

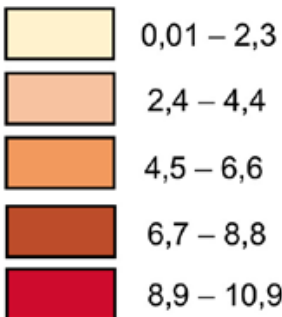

C
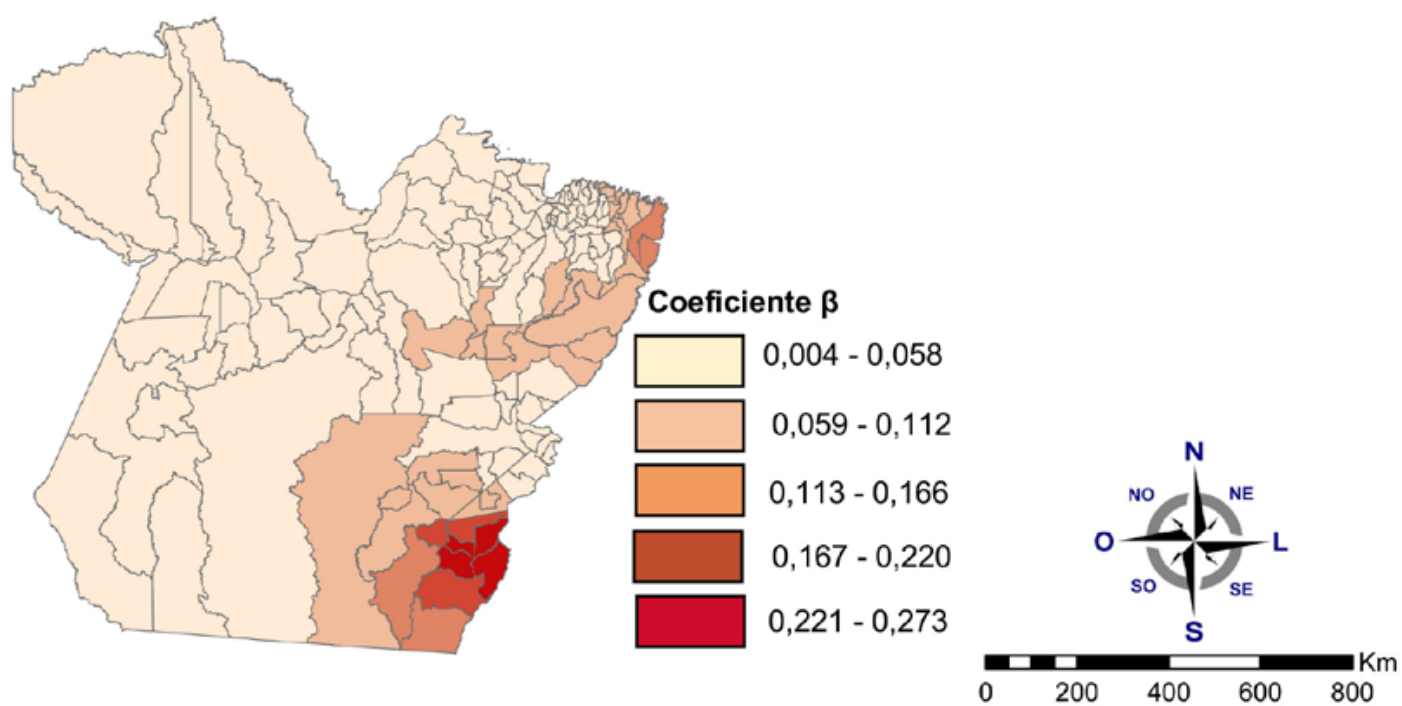

A: \% de crescimento populacional municipal; $B$ : $\mathrm{R}^{2}$ local ajustado; C: Coeficiente $\beta$ do percentual de crescimento populacional municipal com a taxa de incidência do HIV/Aids.

Figura 3. Mapeamento espacial pela regressão geograficamente ponderada do percentual de crescimento populacional municipal com a taxa de incidência do HIV/Aids. Pará, 2020. 


\section{DISCUSSÃO}

Os resultados do presente estudo mostraram que enquanto a incidência do HIV tende a crescer na população a partir de 50 anos de idade no Pará, a de Aids tende a estabilizar-se. A análise espacial mostrou que os municípios do meridional sudeste e sudoeste paraense apresentam as maiores incidências de HIV/Aids, com as maiores zonas de risco espacial formadas por municípios do sudeste paraense e por Belém, enquanto a análise espaço-temporal mostrou que Belém foi a região de maior risco nos anos 20172018. A regressão espacial mostrou que a elevada incidência da epidemia do HIV no sudeste paraense foi fortemente associada ao crescimento populacional desses municípios.

Evidenciou-se ainda que, enquanto o diagnóstico de Aids manteve estabilidade durante todo o período, o de HIV tendeu a crescer. Tal fato pode estar relacionado com a notificação compulsória do diagnóstico do HIV a partir de 2014, e descentralização dos testes de rastreio para o vírus que são executados pelas ESFs ${ }^{21}$. Porém, diferente do observado no continente africano após a universalização do teste anti-HIV e do tratamento antirretroviral com redução da transmissão do vírus, da Aids e da mortalidade por Aids em todas as faixas etárias $^{2324}$, este estudo mostrou aumento da taxa de incidência do HIV e estabilidade na de Aids.

Além da necessidade de expandir a testagem e o tratamento antirretroviral no Pará, impõe-se implementar políticas públicas de conscientização sobre as formas de transmissão e de prevenção do HIV especificamente para esse grupo etário estudado. Estudo realizado na Coreia do Sul com pessoas acima de 65 anos mostrou que aquelas sexualmente ativas, principalmente os homens, mantinham múltipla parceria sexual e não costumavam usar o preservativo. Porém, os que detinham conhecimento sobre os riscos de infecções sexualmente transmissíveis (IST) praticavam sexo mais seguro ${ }^{6}$.

A disparidade de gênero observada em nosso estudo em que a taxa de incidência de Aids apresentou o maior aumento percentual nas mulheres também foi evidenciada em estudos anteriores ${ }^{2,12}$. Nos Estados Unidos, entre 2014 e 2018 decresceu a incidência de
HIV/Aids nos homens com idade igual ou superior a 50 anos, ficando estável entre as mulheres ${ }^{2}$. $\mathrm{Na}$ China a proporção de casos de HIV notificados em mulheres acima de 50 anos saltou passou de 17,83\% em 2010 para 38,10\% em 2016 ${ }^{12}$. A desigualdade de gênero decorrente de fatores biológicos, sociais, culturais, econômicos e religiosos coloca a mulher em situação de maior vulnerabilidade às IST.

A análise espacial evidenciou que os municípios do sudeste e do sudoeste paraense foram os mais impactados pela epidemia do HIV, fenômeno esse diretamente associado ao crescimento populacional. Os municípios do meridional sudeste e do sudoeste paraense apresentam intenso processo de urbanização promovido pela expansão da indústria de mineração, da atividade pecuarista e pela construção de hidrelétricas. O crescimento populacional desses municípios tem-se dado de forma acelerada, desordenada e sem o acompanhamento proporcional de investimento em infraestrutura urbana ${ }^{18,19} . \mathrm{Na}$ África subsaariana, a alta mobilidade da população em busca de melhores condições de vida tem sido apontada como catalisadora da epidemia do $\mathrm{HIV}^{25}$. O crescimento e a densidade populacional está diretamente associado à expansão das IST, recebendo direta influência da desigualdade social, educacional e da renda per capta ${ }^{26}$

Categorizada como segundo maior risco espacial para o HIV, Belém é a cidade do Pará com a maior densidade demográfica ${ }^{16}$. Esses resultados coadunam com estudo feito com idosos no Rio de Janeiro em que as cidades mais populosas do estado, Rio de Janeiro e Niterói, apresentaram as maiores incidências de $\mathrm{Aids}^{27}$.

O baixo investimento em saúde pode também contribuir para o cenário da epidemia do HIV no Pará, estado com uma das mais baixas coberturas de ESF no Brasil (54,5\%), a maioria delas localizandose em zonas urbanas, o que dificulta o acesso aos moradores do interior paraense pela característica geográfica da região e pelo elevado índice de pobreza dos habitantes ${ }^{15,28}$. As ESFs desempenham papel de protagonistas no combate ao HIV/Aids com ações desde a promoção da saúde sexual, prevenção de IST, diagnóstico e acompanhamento dos pacientes ${ }^{29}$. Em Belém, ao final de 2016 foram intensificadas as testagens para o HIV com a descentralização dos 
testes para as $\mathrm{ESFs}^{30}$. Tal fato se coaduna com os resultados da varredura espaço-temporal apontando Belém como região de maior risco no período de 2017-2018.

Por se tratar de estudo ecológico, não se pode inferir causalidade entre a transmissão do HIV com raça ou cor de pele, imigração ou outros fenômenos sociais devido aos fatores confundidores omitidos nesse tipo de análise. Adicionalmente, o estudo foi limitado pelas fichas com dados incompletos, mas em pequeno número, não afetando a análise.

\section{CONCLUSÃO}

O presente estudo evidenciou que enquanto a incidência de Aids em pessoas a partir de 50 anos de idade permaneceu estável no período de 2007 a 2018 no Pará, a incidência de HIV tendeu a crescer entre mulheres e homens. A análise espacial revelou o maior impacto da epidemia nesse grupo etário nos municípios do meridional sudeste paraense e com forte associação ao seu crescimento populacional. A análise espaço-temporal revelou que Belém é o município com maior risco, o que pode estar associado à intensificação das políticas públicas de combate ao HIV a partir de dezembro de 2016 pela Secretaria de Saúde e Meio Ambiente de Belém.

Diante do exposto, sente-se necessidade de maior investimento em políticas públicas de saúde nos eixos sudeste e sudoeste do Pará, Brasil, para o combate ao HIV, contemplando não só a expansão da cobertura da Estratégia da Saúde da Família, mas também investimento em infraestrutura acompanhando o desenvolvimento urbano, de modo a garantir aos cidadãos seu direito universal à saúde.

Editado por: Maria Helena Rodrigues Galvão

\section{REFERÊNCIAS}

1. Brasil. Ministério da Saúde, Secretaria de Vigilância em Saúde, Departamento de Doenças de Condições Crônicas e Infecções Sexualmente Transmissíveis (DCCI). Boletim Epidemiológico HIV/Aids. Brasília, DF: MS; 2019. Disponível em: http://www.aids. gov.br/pt-br/pub/2019/boletim-epidemiologico-dehivaids-2019.

2. Centers for Disease Control and Prevention. HIV and older americans. Atlanta: CDC; 2020. Disponível em: https://www.cdc.gov/hiv/pdf/group/age/ olderamericans/cdc-hiv-older-americans.pdf.

3. Haddad N, Robert A, Popovic N, Varsaneux O, Edmunds M, Jonah L, et al. Newly diagnosed cases of HIV in those aged 50 years and older and those less than 50: 2008-2017. Can Commun Dis Rep. 2019;45(11):283-8. Disponível em: https://doi. org/10.14745/ccdr.v45i11a02.

4. Tavoschi L, Diasa JG, Pharris A. New diagnoses amongo adult aged 50 years or older in 31 europeans countries, 2004-15: an analysis of surveillance data. Lancet HIV. 2017;4(11):514-21. Disponível em: https://doi.org/10.1016/S2352-3018(17)30155-8.

5. Joint United Nations Programme on HIV/AIDS. People aged 50 years and older [Internet]. Genebra: UNAIDS; 2014. [acesso em 23 out. 2020]. Disponível em: https://www.unaids.org/sites/default/files/ media_asset/12_Peopleaged50yearsandolder.pdf.

6. Kim HY, Choe HS, Lee DS, Yoo JM, Lee SJ. Sexual behavior and sexually transmitted infection in the elderly population of South Korea. Investig Clin Urol. 2019;60(3):202-9. Disponível em: https://doi. org/10.4111/icu.2019.60.3.202.

7. Oraka E, Mason S, Xia M. Too old to test? Prevalence and correlates of HIV testing among sexually active older adults. J Gerontol Soc Work 2018;61(4):460-70. Disponível em: https://doi.org/10.1080/01634372.20 18.1454565 .

8. Tavoschi L, Dias JG, Pharris A. New HIV diagnoses among adults aged 50 years or older in 31 European countries, 2004-15: an analysis of surveillance data. Lancet HIV. 2017;4(11):514-21. Disponível em: https://doi.org/10.1016/S2352-3018(17)30155-8.

9. United Nations. Global Issues. Ageing [Internet]. Geneva: UN; 2020. Disponível em: https://www. un.org/en/sections/issues-depth/ageing/index.html.

10. Global Burden of Diseases HIV. Global, regional, and national incidence, prevalence, and mortality of HIV, 1980-2017, and forecasts to 2030, for 195 countries and territories: a systematic analysis for the Global Burden of Diseases, Injuries, and Risk Factors Study 2017. Lancet HIV. 2019;6(12):e831-e859. Disponível em: https://doi.org/10.1016/S2352-3018(19)30196-1. 
11. Rebolledo EAS, Chiaravalloti F, Giatti LL. Experiencias, beneficios y desafíos del uso de geoprocesamiento para el desarrollo de la atención primaria de salud. Rev Panam Salud Publica. 2018;42:1-10. Disponível em: https://doi. org/10.26633/RPSP.2018.153

12. Chen FF, Guo W, Qin QQ, Cai C, Cui Y. Spatialtemporal distribution of newly detected HIV/ AIDS cases among aged 15 years or older women in China, 2010-2016. Zhonghua Liu Xing Bing Xue Za Zhi. 2018;39(6):739-44. Disponível em: https://doi. org/10.3760/cma.j.issn.0254-6450.2018.06.009 .

13. Xing J, Li YG, Tang W, Guo W, Ding Z, Ding G, et al. HIV/AIDS epidemic among older adults in China during 2005-2012: results from trend and spatial analysis. Clin Infect Dis. 2014;59(2):53-60. Disponível em: https://doi.org/10.1093/cid/ciu214.

14. Nogueira JA, Silva AO, Sá LR, Almeida SA, Monroe AA, Villa TCS. AIDS in adults 50 years of age and over: characteristics, trends and spatial distribution of the risk. Rev Latinoam Enferm. 2014;22(3):355-63. Disponível em: https://doi.org/10.1590/0104-1169.3327.2424.

15. Neves RG, Flores TR, Duro SMS, Nunes BP, Tomasi E. Tendência temporal da cobertura da Estratégia Saúde da Família no Brasil, regiões e Unidades da Federação, 2006-2016. Epidemiol Serv Saúde. 2018;27(3):e2017170. Disponível em: https://doi. org/10.5123/s1679-49742018000300008.

16. Instituto Brasileiro de Geografia e Estatística. Índice de Desenvolvimento Humano: Pará, 2010 [Internet]. Rio de Janeiro: IBGE; 2017. [acesso em 23 out. 2020]. Disponível em: https://cidades.ibge.gov.br/brasil/pa/ pesquisa $/ 37 / 0$ ?ano $=2010$.

17. Costa SMF, Carmo MBS, Barja PR. The urban hierarchy at the delta of the Amazon River and the importance of small cities. Rev Bras Gest Urbana. 2019;11:e20180014. Disponível em: https://doi. org/10.1590/2175-3369.011.001.AO09 .

18. Richards P, VanWey L. Where deforestation leads to urbanization: how resource extraction is leading to urban growth in the Brazilian Amazon. Ann Assoc Am Geogr. 2015;105(4):806-23. Disponível em: https://doi.org/10.1080/00045608.2015.1052337.

19. Fundação Amazônia de Amparo a Estudos e Pesquisas. Anuário Estatístico do Pará: 2019 [Internet]. Belém: FAPESPA; 2019 [acesso em 23 out. 2020]. Disponível em: http://www.fapespa.pa.gov.br/ sistemas/anuario2019/tabelas/demografia/tab-1.2populacao-por-faixa-etaria-2011-a-2015.htm .

20. Joint United Nations Programme on HIV/AIDS. Report on the global HIV/AIDS epidemic: June 1998. [sem local]: [sem editora]; 1988. Disponível em: http:// data.unaids.org/pub/report/1998/19981125_global_ epidemic_report_en.pdf
21. Brasil. Portaria no 1.271, de 6 de junho de 2014. Define a Lista Nacional de Notificação Compulsória de doenças, agravos e eventos de saúde pública nos serviços de saúde públicos e privados em todo o território nacional, nos termos do anexo, e dá outras providências. Diário Oficial da União. 06 de jun. 2014. Disponível em: http://bvsms.saude.gov.br/bvs/ saudelegis/gm/2014/prt1271_06_06_2014.html.

22. Kim HJ, Fay MP, Feuer EJ, Midthune DN. Permutation tests for joinpoint regression with applications to cancer rates. Stat Med. 2000;19(3):33551. Disponível em: https://doi.org/10.1002/(SICI)10970258(20000215)19:3<335::AID-SIM336>3.0.CO;2-Z.

23. Kulldorff M, Nagarwalla N. Spatial disease clusters: detection and inference. Stat Med. 1995;14:799-810. Disponível em: https://doi.org/10.1002/sim.4780140809 .

24. Havlir D, Lockman S, Ayles H, Larmarange J, Chamie G, Gaolathe T, et al. What do the Universal Test and Treat trials tell us about the path to HIV epidemic control? J Int AIDS Soc. 2020;23(2):e25455. Disponível em: https://doi.org/10.1002/jia2.25455.

25. Dobra A, Bärnighausen T, Vandormael A, Tanser F. Space-time migration patterns and risk of HIV acquisition in rural South Africa. AIDS. 2020;31(1):137-45. Disponível em: https://doi. org/10.1097/QAD.0000000000001292.

26. Patterson-Lomba O, Goldstein E, Gómez-Liévano A, Castillo-Chavez C, Towers S. Per-capita Incidence of Sexually Transmitted Infections Increases Systematically with Urban Population Size: a cross-sectional study. Sex Transm Infect. 2015;91(8):610-14. Disponível em: https://doi. org/10.1136/sextrans-2014-051932.

27. Rodrigues NCP, Almeida AS, Braga JU, O’Dwyer G, Apratto Jr. PC, Daumas RP, et al. Spatial dynamics of AIDS incidence in the elderly in Rio de Janeiro, Brazil, 19972011. Cad Saúde Pública. 2015;31(8):1721-31. Disponível em: https://doi.org/10.1590/0102-311X00152914.

28. Garnelo L, Lima JG, Rocha ESC, Herkrath FJ. Acesso e cobertura da Atenção Primária à Saúde para populações rurais e urbanas na região norte do Brasil. Saúde Debate. 2018;42(spec 1):81-99. Disponível em: https://doi.org/10.1590/0103-11042018s106.

29. Melo EA, Maksud I, Agostini R. Cuidado, HIV/Aids e atenção primária no Brasil: desafio para a atenção no Sistema Único de Saúde? Rev Panam Salud Publica. 2018;42:e151. Disponível em: https://doi. org/10.26633/RPSP.2018.151.

30. Belém. Prefeitura Municipal. Plano Plurianual - PPA 2014-2017 [Internet]. Belém: PMB; 2014 [acesso em 23 out. 2020]. Disponível em: http://www.belem.pa.gov. br/transparencia/?page_id=602\#15109170721448789 a 5 af- 8 c $2 b$ 